Pacific

Journal of

Mathematics

NOTES ON THE STRUCTURE CONSTANTS OF HECKE ALGEBRAS OF INDUCED REPRESENTATIONS OF FINITE CHEVALLEY GROUPS

Charles W. Curtis

Volume 279 No. 1-2

December 2015 


\title{
NOTES ON THE STRUCTURE CONSTANTS OF HECKE ALGEBRAS OF INDUCED REPRESENTATIONS OF FINITE CHEVALLEY GROUPS
}

\author{
Charles W. Curtis \\ This paper is dedicated to the memory of Robert Steinberg. \\ This paper contains an algorithm for the structure constants of the Hecke \\ algebra of a Gelfand-Graev representation of a finite Chevalley group.
}

\section{Introduction}

Let $G$ be a Chevalley group over a finite field $k=F_{q}$ of characteristic $p$ (as in [Chevalley 1955] or [Steinberg 1968]). Let $B$ be a Borel subgroup of $G$ with $U=O_{p}(B)$ (the unipotent radical of $B$ ), and let $T$ be a maximal torus such that $B=U T$. Let $W$ be the Weyl group of $G$. Then $W$ is a finite Coxeter group with distinguished generators $S=\left\{s_{1}, \ldots, s_{n}\right\}$.

Let $\Phi$ be the root system associated with $W$, with $\left\{\alpha_{1}, \ldots, \alpha_{n}\right\}$ the set of simple roots corresponding to the generators $s_{i} \in S$, and $\Phi_{ \pm}$the set of positive roots (respectively, negative roots) associated with them. For each root $\alpha$, let $U_{\alpha}$ be the root subgroup of $G$ corresponding to it. The subgroup $U$ is generated by the root subgroups $U_{\alpha}, \alpha>0$.

From [Steinberg 1968, §3], the Chevalley group $G$ has a $B, N$-pair, with Borel subgroup $B, N$ the subgroup generated by all elements $w_{\alpha}(t)$, and $B \cap N$ equal to $T$, the subgroup generated by all elements $h_{\alpha}(t)$ (see the definitions of $w_{\alpha}(t)$ and $h_{\alpha}(t)$ in Section 2). Then $N / T \cong W$. (If the field $k$ contains more than three elements, then $N$ is the normalizer $N=N_{G}(T)$; see [Steinberg 1968, p. 36]).

By the Bruhat decomposition, the $(U, U)$-double cosets are parametrized by the elements of $N$, while the $(B, B)$-double cosets are parametrized by the elements of $W$.

We consider induced representations $\gamma$ of the form $\psi^{G}$, for a linear representation $\psi$ of $U$. Let

$$
e=|U|^{-1} \sum_{u \in U} \psi\left(u^{-1}\right) u
$$

MSC2010: 20G40.

Keywords: representation theory, finite Chevalley groups. 
be the primitive idempotent affording $\psi$ in the group algebra $\mathbb{C} U$ of $U$ over the field of complex numbers. Then $\gamma=\psi^{G}$ is afforded by the left $\mathbb{C} G$-module $\mathbb{C} G e$. The Hecke algebra of $\gamma$ is the subalgebra $H=e \mathbb{C} G e$ of $\mathbb{C} G$, and is isomorphic to $\left(\text { End }_{\mathbb{C} G} \mathbb{C} G e\right)^{\circ}$. These representations and their Hecke algebras were first investigated by Gelfand and Graev [1962a; 1962b]. In particular, they introduced the important class of Gelfand-Graev representations of $G$, which are the induced representation $\psi^{G}$, for a linear representation $\psi$ of $U$ in general position, that is, $\psi \mid U_{\alpha_{i}} \neq 1$ for each simple root subgroup $U_{\alpha_{i}}, 1 \leq i \leq n$, and $\psi \mid U_{\alpha}=1$ for each positive and not simple root $\alpha$.

It is known (see [Gelfand and Graev 1962b] for the case of $G=\mathrm{SL}_{n}(k)$ for a finite field $k$, and [Steinberg 1968, Theorem 49] for the general case) that the Hecke algebra $H$ of a Gelfand-Graev representation is a commutative algebra, so that a Gelfand-Graev representation is multiplicity-free.

A basis for the Hecke algebra $H$ of a Gelfand-Graev representation $\psi^{G}$ is given by the nonzero elements of the form ene with $n \in N$. The standard basis elements are the nonzero elements of the form $c_{n}=\operatorname{ind}(n)$ ene, where ind $(n)=\left|U: n U n^{-1} \cap U\right|$. The structure constants for the standard basis elements, defined by the formulas

$$
c_{\ell} c_{m}=\sum_{n}\left[c_{\ell} c_{m}: c_{n}\right] c_{n},
$$

with $\ell, m, n \in N^{*}$, are algebraic integers (here $N^{*}$ is the set of elements $n \in N$ such that ene $\neq 0$ ).

The structure constants of $H$ are given by the formula

$$
\left[c_{\ell} c_{m}: c_{n}\right]=\sum_{u \ell u_{1}=n v m^{-1} \in U \ell U \cap n U_{m^{-1}} m^{-1}} \psi\left(\left(u u_{1}\right)^{-1} v\right),
$$

by [Curtis and Reiner 1981, Proposition 11.30], and the fact that $U \ell U \cap n U_{m^{-1}} m^{-1}$ is a set of representatives of the left $U$-cosets in $U \ell U \cap n U_{m^{-1}} m^{-1} U$. As in [Curtis 1988; 2009], $U_{n}=U \cap n U_{-} n^{-1}$ for $n \in N$. The structure constants are exponential sums involving the linear character $\psi$ of $U$ and combinatorial information about multiplication and intersections patterns of $(U, U)$-double cosets. The latter information is also given at least partially for the algebraic group $G(\bar{k})$ over the algebraic closure $\bar{k}$ of $k$ corresponding to $G$, with some questions about the geometry not completely settled at this time. A main result in the paper is an algorithm given in Section 4 for the solutions $\left(u, u_{1}, v\right)$ of the equation $u \ell u_{1}=n v m^{-1}$ in the formula above, so that in some sense the structure constants are computable. The approach taken here is based on the theory of cells $U_{\tau}$ developed in [Curtis 1988; 2009]. The algorithm for the solutions of the equations is a refined version of an algorithm for them given in [Curtis 2009, Theorem 2.1]. At the end of Section 4, some problems for further research are mentioned. 
In case $\psi^{G}$ is a Gelfand-Graev representation, the values of the irreducible representations of the commutative semisimple algebra $H$ on standard basis elements are obtained as eigenvalues of matrices giving the regular representation of $H$ and whose entries are the structure constants $\left[c_{\ell} c_{m}: c_{n}\right.$ ]; see [Curtis 2009, Proposition 1.1].

Formulas for the structure constants based on different algorithms and a different set of representatives of the cosets of $U$ were obtained by Simion [2015].

The irreducible representations of $H$ were obtained in [Curtis 1993] using the results of Deligne and Lusztig [1976] on representations of $G$ defined on the $\ell$-adic cohomology of locally closed subsets of the algebraic group $G(\bar{k})$ with Frobenius endomorphism $F$ on which the finite group $G$ acts. The formulas for the irreducible representations of $H$ in [Curtis 1993] involve a homomorphism of algebras $f_{T}: H \rightarrow \mathbb{C} T$ for each $F$-stable maximal torus $T$ of $G$, proved using the character formula of Deligne and Lusztig [1976] for the virtual representations $R_{T, \theta}$. The homomorphisms $f_{T}$ provide an approach to the representations of $H$, and are of independent interest (see [Bonnafé and Kessar 2008]).

A combinatorial approach to the representations of $H$ based on the structure constants of the Hecke algebra $H$ and the internal structure of the finite Chevalley group $G$ is a main objective of this paper.

Two final sections contain examples in which a combinatorial construction of the homomorphisms $f_{T}$ is obtained. These include the Bessel functions over finite fields of Gelfand and Graev [1962a], for the groups $\mathrm{SL}_{2}(k)$ and $k$ a finite field of odd characteristic, and a construction of the homomorphisms $f_{T}: H \rightarrow \mathbb{C} T$ for the split torus $T$ in a general Chevalley group.

\section{Background and preliminary results}

For each root $\alpha$, there is a homomorphism (see [Steinberg 1968, page 46]) $\varphi=\varphi_{\alpha}$ : $\mathrm{SL}_{2}(k) \rightarrow G$ such that $\varphi$ takes

$$
\left(\begin{array}{ll}
1 & t \\
0 & 1
\end{array}\right) \rightarrow x_{\alpha}(t), \quad\left(\begin{array}{ll}
1 & 0 \\
t & 1
\end{array}\right) \rightarrow x_{-\alpha}(t), \quad\left(\begin{array}{cc}
0 & t \\
-t^{-1} & 0
\end{array}\right) \rightarrow w_{\alpha}(t) \in N, \quad\left(\begin{array}{cc}
t & 0 \\
0 & t^{-1}
\end{array}\right) \rightarrow h_{\alpha}(t) \in T
$$

for all $t \in k$. The elements $w_{\alpha}(t)$ and $h_{\alpha}(t)$ are given by

$$
w_{\alpha}(t)=x_{\alpha}(t) x_{-\alpha}\left(-t^{-1}\right) x_{\alpha}(t), \quad h_{\alpha}(t)=w_{\alpha}(t) w_{\alpha}(1)^{-1},
$$

by [Steinberg 1968, p. 30]. If $w=s_{k} \cdots s_{1}$ is a reduced expression of an element $w \in W$ then $\dot{w}=\dot{s}_{k} \cdots \dot{s}_{1}$, with $\dot{s}_{i}=w_{\alpha_{i}}\left(t_{i}\right)$ for some fixed choice of $t_{i} \in k^{*}=k-\{0\}$, is a representative in $N$ of $w$ which is independent of the choice of the reduced expression chosen, by [Steinberg 1968, Lemma 83, p. 242]. In what follows we assume that representatives $\dot{x} \in N$ of all elements $x \in W$ have been chosen in this way, for a fixed choice of representatives $\dot{s}_{i}$ of the generators $s_{i} \in S$. 
We may assume that

$$
\dot{s}_{k}=\varphi_{\alpha_{k}}\left(\left(\begin{array}{rr}
0 & 1 \\
-1 & 0
\end{array}\right)\right)
$$

for a simple root $\alpha_{k}, 1 \leq k \leq n$.

Using the homomorphisms $\varphi_{\alpha}$, we obtain the so-called $\mathrm{SL}_{2}$-IDENTITY:

$$
\dot{s}_{k}^{-1} x_{\alpha_{k}}(t) \dot{s}_{k}=x_{\alpha_{k}}\left(-t^{-1}\right) \dot{s}_{k} h_{\alpha_{k}}(t) x_{\alpha_{k}}\left(-t^{-1}\right),
$$

for a simple root $\alpha_{k}$ and $t \in k^{*}$ (cf. [Curtis 2009, Lemma 2.1]).

As in [Deodhar 1985], a subexpression $\tau$ of a fixed reduced expression $w=$ $s_{k} \cdots s_{1}$ is a sequence $\tau=\left(\tau_{k}, \ldots, \tau_{1}, \tau_{0}\right)$ of elements of $W$ such that $\tau_{i} \tau_{i-1}^{-1} \in\left\{1, s_{i}\right\}$ for $i=1, \ldots, k$ and $\tau_{0}=1$. Then the set of terminal elements $\tau_{k}$ of subexpressions of $w=s_{k} \cdots s_{1}$ coincides with the set of elements $x \in W$ such that $x \leq w$ in the Chevalley-Bruhat order. In what follows, the length of an element $w \in W$ in terms of the generators $s_{i} \in S$ is denoted by $\ell(w)$. A subexpression $\tau=\left(\tau_{k}, \ldots, \tau_{1}, \tau_{0}\right)$ is called a $K$-sequence relative to the triple $w=s_{k} \cdots s_{1}, x, y$ of elements of $W$ if it satisfies conditions (2.10)(a-c) of [Kawanaka 1975]. It is understood that a $K$ sequence for the triple $(w, x, y)$ is always given with reference to a fixed reduced expression $w=s_{k} \cdots s_{1}$. Let $J_{\tau}=\left\{j: \tau_{j} \tau_{j-1}^{-1}=s_{j}\right\} \cup\{0\}$. Then the defining conditions for a $K$-sequence state that $\tau_{k} x=y$ and

$$
\ell\left(s_{p} \tau_{j} x\right)<\ell\left(\tau_{j} x\right)
$$

for each $j \in J_{\tau}$ and $p$ in the interval between $j$ and the next element in $J_{\tau}$ (or simply all $p>j$ if $j$ is the maximal element of $J_{\tau}$ ). For each $K$-sequence $\tau$, set

$$
J_{\tau}^{-}=\left\{j \in J_{\tau}: \ell\left(s_{j} \tau_{j^{\prime}} x\right)<\ell\left(\tau_{j^{\prime}} x\right)\right\}
$$

where $j^{\prime} \in J_{\tau}$ is the predecessor of $j$, and define a pair of nonnegative integers by

$$
a(\tau)=\left|J_{\tau}^{-}\right|, b(\tau)=k-\left|J_{\tau}\right|+1=\operatorname{card}\left\{j>0: \tau_{j} \tau_{j-1}^{-1}=1\right\} .
$$

For each element $w \in W$, let $U_{w}=U \cap{ }^{w} U_{-}$where $U_{-}={ }^{w_{0}} U$ and $w_{0}$ is the element of maximal length in $W$. Then $U=U_{w} U_{w w_{0}}$ and $B w B=U_{w} \dot{w} B$, in both cases with uniqueness of expression. Let $w=s_{k} \ldots s_{1}$ be a reduced expression of $w \in W$. Then $U_{w}=U_{\alpha_{k}} \dot{s}_{k} U_{s_{k-1} \ldots s_{1}} \dot{s}_{k}^{-1}$ with uniqueness of expression. An element of $U_{w}$ expressed in this way, for a fixed reduced expression of $w$, is said to be in standard form (see [Deodhar 1985, Lemma 2.2]), and can be assigned coordinates in the field $k$.

Let $w, x, y$ be elements of $W$, and $\dot{w}, \dot{x}, \dot{y}$ corresponding elements of $N$. Let

$$
U(w, x, y)=\left\{u \in U_{w}: u \dot{w} B \cap \dot{y} U_{x^{-1}} \dot{x}^{-1} \neq \varnothing\right\} .
$$

Then $U(w, x, y)$ is independent of the choice of representatives $\dot{w}, \dot{x}, \dot{y}$ of $w, x, y$ in $N$. Moreover, $U_{w} \dot{w} B \cap \dot{y} U_{x^{-1}} \dot{x}^{-1}$ is a set of representatives of the left $B$-cosets 
in $B w B \cap y(B x B)^{-1}$, and its cardinality is the structure constant $\left[e_{w} e_{x}: e_{y}\right]$ of the standard basis elements $e_{w}, e_{x}, e_{y}$, for $w, x, y \in W$, in the Iwahori Hecke algebra.

The $K$-sequences were first applied by Kawanaka to prove the following result [Kawanaka 1975, Lemma 2.14b]. For a finite Chevalley group $G$ over $k=F_{q}$ the nonzero structure constants of the Iwahori Hecke algebra are given by the formula

$$
\left[e_{w} e_{x}: e_{y}\right]=\left|B \dot{w} B \cap \dot{y} U_{x^{-1}} \dot{x}^{-1}\right|=|U(w, x, y)|=\sum_{\tau} q^{a(\tau)}(q-1)^{b(\tau)}
$$

where the sum is taken over all $K$-sequences $\tau$ for $w, x, y$, and $a(\tau)$ and $b(\tau)$ are the nonnegative integers defined above.

As a consequence, it follows that $U(w, x, y) \neq \varnothing$ if and only if there exist $K$-sequences for $w, x, y$ (see also [Borel and Tits 1972, Remark 3.19], where the conditions are stated in a different way).

In [Curtis 1988] a geometric version of Kawanaka's formula was proved. It states that $U(w, x . y)$, viewed as a subset of the algebraic group $G(\bar{k})$, is a disjoint union of subsets $U_{\tau}$, which we shall call (in this paper) cells. The cells $U_{\tau}$ are subsets of $G(\bar{k})$ parametrized by $K$-sequences $\tau$ for $w, x, y$ relative to a fixed reduced expression of the element $w$, with corresponding subsets $U_{\tau}$, also called cells (defined in [Curtis 1988]), in the finite Chevalley group $G=G(k)$ (see Lemma 3.3 below for a review of the definition of cells). The result extends Deodhar's decomposition ([Deodhar 1985], and [Curtis 2009, §4]) of the intersection $B y B \cap B_{-} x B$, viewed as subsets of the flag variety $G / B$ in the algebraic group $G(\bar{k})$, with $B_{-}$the Borel subgroup opposite to $B$. Each cell $U_{\tau}$ is isomorphic (in bijective correspondence as a set, or isomorphic as a variety in $G(\bar{k}))$ to a product,

$$
U_{\tau} \cong \prod_{\alpha} U_{\alpha} \times \prod_{\beta} U_{\beta}^{*}
$$

for certain subsets $\{\alpha\}$ and $\{\beta\}$ of cardinalities $a(\tau)$ and $b(\tau)$ of the positive root subgroups determined by $\tau$ and where $U_{\beta}^{*}$ is the set of nonidentity elements in $U_{\beta}$. From the decomposition of $U(w, x, y)$ as a union of cells $U_{\tau}$, it follows that $U_{w} \dot{w} B \cap \dot{y} U_{x^{-1}} \dot{x}^{-1}$ can be identified with the set of triples $(u, b, v)$ with $u \in U_{\tau}$ for some $\tau, b \in B$, and $v \in U_{x^{-1}}$ satisfying the equation $u \dot{w} b=\dot{y} v \dot{x}^{-1}$ with $b$ and $v$ uniquely determined by $u$ by [Curtis 2009, Lemma 2.4].

\section{Relations between cells}

Let $\ell, m, n$ in $N^{*}$ correspond to elements $w, x, y$ in $W$. Then $\ell, m, n$ are multiples by elements of $T$ of representatives $\dot{w}, \dot{x}, \dot{y}$ in $N$ determined as above. The set $U_{\ell} \ell U \cap n U_{m^{-1}} m^{-1}$ will be obtained by an algorithm based on a fixed reduced expression $w=s_{k} \cdots s_{1}$ of the element $w \in W$ in terms of the generators $s_{i} \in S$, and the theory of cells $U_{\tau}$ associated with $K$-sequences $\tau$ for $w, x, y$. 
As the cells $U_{\tau}$ are contained in the set $U(w, x, y)$ each element $u \in U_{\tau}$ satisfies a structure equation

$$
u \dot{w} b=\dot{y} v \dot{x}^{-1}
$$

with $b \in B$ and $v \in U_{x^{-1}}$. The subgroup $B$ is a semidirect product $B=U T$, so one has $b=u_{1} s$ with $u_{1} \in U, s \in T$, and it will be important to keep track of these factors in the discussion to follow.

In this section, it will be shown how elements $u \in U_{\tau} \subseteq U(w, x, y)$, with $\tau=\left(\tau_{k}, \ldots, \tau_{1}, \tau_{0}\right)$ a $K$-sequence for $w, x, y$, are related to elements $u^{\prime}$ in cells $U_{\tau^{\prime}}$ with $\tau^{\prime}=\left(\tau_{k-1}, \ldots, \tau_{1}, \tau_{0}\right)$ a $K$-sequence for $s_{k-1} \cdots s_{1}, x^{\prime}, y^{\prime}$, and how the structure equations for $u$ and $u^{\prime}$ are related. We keep in mind that $U(w, x, y) \neq \varnothing$ if and only if there exist $K$-sequences for $w, x, y$.

Lemma 3.1. Let $\tau=\left(\tau_{k}, \ldots, \tau_{1}, \tau_{0}\right)$ be a $K$-sequence for $w, x, y$ for $k \geq 1$, and consider $\tau^{\prime}=\left(\tau_{k-1}, \ldots, \tau_{0}\right)$.

(i) $\tau^{\prime}$ is a $K$-sequence for $s_{k}^{-1} w, x, s_{k}^{-1} y$ if $\tau_{k} \tau_{k-1}^{-1}=s_{k}$ and $\ell\left(s_{k} y\right)<\ell(y)$.

(ii) $\tau^{\prime}$ is a $K$-sequence for $s_{k}^{-1} w, x, y$ if $\tau_{k} \tau_{k-1}^{-1}=1$ and $\ell\left(s_{k} y\right)<\ell(y)$.

(iii) $\tau^{\prime}$ is a $K$-sequence for $s_{k}^{-1} w, x, s_{k}^{-1} y$ if $\ell\left(s_{k} y\right)>\ell(y)$ and $\tau_{k} \tau_{k-1}^{-1}=s_{k}$.

It is understood that $\tau_{0}=1$ is a $K$-sequence for $(1, x, x)$ and that $a\left(\tau_{0}\right)=b\left(\tau_{0}\right)=0$. These sets of conditions are the only possibilities for $\tau^{\prime}$ to be a $K$-sequence, and one of them must occur.

We first note that either $\ell\left(s_{k} y\right)<\ell(y)$ or $\ell\left(s_{k} y\right)>\ell(y)$, since either $y^{-1}\left(\alpha_{k}\right) \in \Phi_{+}$ or $y^{-1}\left(\alpha_{k}\right) \in \Phi_{-}$. The proof then follows immediately from the definition of $K$-sequence (see the proof of Lemma 2.14 of [Kawanaka 1975]). For example, we verify that the condition $\ell\left(s_{k} y\right)>\ell(y)$ implies $\tau_{k} \neq \tau_{k-1}$, and hence $\tau_{k} \tau_{k-1}^{-1}=s_{k}$. Otherwise $\tau_{k}=\tau_{k-1}, \tau_{k-1} x=y$, and $k \notin J_{\tau}$. This implies that $\ell\left(s_{k} \tau_{k-1} x\right)<\ell\left(\tau_{k-1} x\right)$ by a defining property of $K$-sequences, and hence $\ell\left(s_{k} y\right)<\ell(y)$, contrary to assumption.

The next result is background for the relation between cells $U_{\tau}$ and $U_{\tau^{\prime}}$, with $\tau$ and $\tau^{\prime}$ as in the preceding lemma. It is a version of Lemma 2.3 of [Curtis 2009]. (Parts (i) and (ii) were misstated in that article and are corrected here. We also take the opportunity to correct the statement on page 220 of [Curtis 2009] that the cells $U_{\tau}$ are invariant under conjugation by elements of $T$; this was not shown there.)

Lemma 3.2. Let $w=s_{k} \cdots s_{1}$ be a reduced expression with $k \geq 1$ and let $x, y \in W$. Then $U(w, x, y)$ is either empty or is related to sets $U\left(s_{k}^{-1} w, x^{\prime}, y^{\prime}\right)$, with $x^{\prime}$ and $y^{\prime}$ depending on the $K$-sequence $\tau$ associated with $w, x, y$ as follows.

(i) Let $\ell\left(s_{k} y\right)<\ell(y)$ and assume $\tau_{k} \tau_{k-1}^{-1}=s_{k}$. Then $\dot{s}_{k} U_{s_{k}^{-1}} \dot{s}_{k}^{-1} \cap U(w, x, y)$ is either empty or

$$
\dot{s}_{k} U_{s_{k}^{-1}} \dot{s}_{k}^{-1} \cap U(w, x, y)=\dot{s}_{k} U\left(s_{k}^{-1} w, x, s_{k}^{-1} y\right) \dot{s}_{k}^{-1} .
$$


(ii) Let $\ell\left(s_{k} y\right)<\ell(y)$ and assume $\tau_{k} \tau_{k-1}^{-1}=1$. Then the part $U(w, x, y)^{b}$ of $U(w, x, y)$ not in $\dot{s}_{k} U_{s_{k}^{-1}} w^{-1}$ consists of the elements $u=x_{\alpha_{k}}(t) \dot{s}_{k} \tilde{u} \dot{s}_{k}^{-1}$, with $x_{\alpha_{k}}(t) \in U_{\alpha_{k}}^{*}$ and $\tilde{u} \in U_{s_{k}^{-1} w}$ such that $\pi\left(x_{\alpha_{k}}\left(-t^{-1}\right) \tilde{u}\right) \in U\left(s_{k}^{-1} w, x, y\right)$, and $t \in k^{*}$; here $\pi$ is the projection $\pi: U \rightarrow U_{s_{k}^{-1} w}$ accompanying the decomposition $U=U_{s_{k}^{-1} w} U_{s_{k}^{-1} w w_{0}}$. The map

$$
u=x_{\alpha_{k}}(t) \dot{s}_{k} \tilde{u} \dot{s}_{k}^{-1} \rightarrow \pi\left(x_{\alpha_{k}}\left(-t^{-1}\right) \tilde{u}\right)
$$

from $U(w, x, y)^{b}$ to $U\left(s_{k}^{-1} w, x, y\right)$ is surjective. There is a bijection of sets $U(w, x, y)^{b} \cong U_{\alpha_{k}}^{*} \times U\left(s_{k}^{-1} w, x, y\right)$.

(iii) Let $\ell\left(s_{k} y\right)>\ell(y)$ and $\tau_{k} \tau_{k-1}^{-1}=s_{k}$. Then

$$
U(w, x, y)=U_{\alpha_{k}} \dot{s}_{k} U\left(s_{k}^{-1} w, x, s_{k}^{-1} y\right) \dot{s}_{k}^{-1}
$$

and there is a bijection of sets $U(w, x, y) \cong U_{\alpha_{k}} \times U\left(s_{k}^{-1} w, x, s_{k}^{-1} y\right)$.

The proof is included in the proof of Lemma 2.3 of [Curtis 2009].

Lemma 3.3. Let $w, x, y$ be elements of $W$ and let $w=s_{k} \cdots s_{1}$ be a reduced expression for $w$. Let $\tau=\left(\tau_{k}, \ldots \tau_{1}, \tau_{0}\right)$ be a $K$-sequence for $w, x, y$ with $\tau_{0}=1$, and let $U_{\tau}$ be the corresponding cell, viewed as a subset of $U(w, x, y) \subseteq U_{w}$. Let $\tau^{\prime}=\left(\tau_{k-1}, \ldots, \tau_{1}, \tau_{0}\right)$ be a $K$-sequence for $s_{k-1} \cdots s_{1}, x^{\prime}, y^{\prime}$ as in one of the cases in Lemma 3.1, and let $U_{\tau^{\prime}}$ be the corresponding cell in $U\left(s_{k-1} \cdots s_{1}, x^{\prime}, y^{\prime}\right)$. The construction of the cell $U_{\tau}$ from $U_{\tau^{\prime}}$, reviewed below, defines a surjective map of sets $\lambda: U_{\tau} \rightarrow U_{\tau^{\prime}}$. Let $U_{\tau}(\bar{k})$ and $U_{\tau^{\prime}}(\bar{k})$ be the corresponding cells in the algebraic group $G(\bar{k})$ over the algebraic closure $\bar{k}$ of $k$. Then the map $\lambda: U_{\tau}(\bar{k}) \rightarrow U_{\tau^{\prime}}(\bar{k})$, defined as in part (i), is a surjective morphism of algebraic sets, defined over $k$.

The construction of $U_{\tau}(\bar{k})$ from $U_{\tau^{\prime}}(\bar{k})$ was given in the three cases of Lemma 3.1 in the proof of Theorem 1.6 of [Curtis 1988] and in [Curtis 2009, page 220], and will be reviewed here in the case of the algebraic group $G(\bar{k})$. We abbreviate $U_{\tau}(\bar{k})$ to $U_{\tau}$, etc.

(i) $\tau_{k} \tau_{k-1}^{-1}=s_{k}$ and $\ell\left(s_{k} y\right)<\ell(y)$. In this case, we have $U_{\tau} \subseteq \dot{s}_{k} U_{s_{k}^{-1}} \dot{s}_{k}^{-1}$ and $U_{\tau^{\prime}} \subseteq U\left(s_{k}^{-1} w, x, s_{k}^{-1} y\right)$, and one has $U_{\tau^{\prime}}=\dot{s}_{k}^{-1} U_{\tau} \dot{s}_{k}$. The map $\lambda: u \rightarrow \dot{s}_{k}^{-1} u \dot{s}_{k}$ is clearly a surjective morphism from $U_{\tau}$ to $U_{\tau^{\prime}}$ and is defined over $k$ because $\dot{s}_{k}$ belongs to the finite Chevalley group $G(k)$.

(ii) $\tau_{k} \tau_{k-1}^{-1}=1$ and $\ell\left(s_{k} y\right)<\ell(y)$. This time $U_{\tau}$ is in the part of $U(w, x, y)$ which is not contained in $\dot{s}_{k} U_{s_{k}^{-1}} \dot{s}_{k}^{-1}$ and consists of the elements $x_{\alpha_{k}}\left(t_{k}\right) \dot{s}_{k} \tilde{u} \dot{s}_{k}^{-1}$ such that $t_{k} \neq 0, \tilde{u} \in U_{s_{k}^{-1} w}$ and $\pi\left(x_{\alpha_{k}}\left(-t_{k}^{-1}\right) \tilde{u}\right) \in U_{\tau^{\prime}}$, where $\pi$ is the projection $U \rightarrow U_{s_{k}^{-1} w}$ associated with the factorization $U=U_{s_{k}^{-1} w} U_{s_{k}^{-1} w w_{0}}$. The map

$$
\lambda: x_{\alpha_{k}}\left(t_{k}\right) \dot{s}_{k} \tilde{u} \dot{s}_{k}^{-1} \rightarrow \pi\left(x_{\alpha_{k}}\left(-t_{k}^{-1}\right) \tilde{u}\right)
$$

is a surjective morphism defined over $k$ from $U_{\tau}$ to $U_{\tau^{\prime}}$. 
(iii) $\tau_{k} \tau_{k-1}^{-1}=s_{k}$ and $\ell\left(s_{k} y\right)>\ell(y)$. In this situation, we have $U_{\tau}=U_{\alpha_{k}} \dot{s}_{k} U_{\tau^{\prime}} \dot{s}_{k}^{-1}$, in terms of the factorization: $U_{w}=U_{\alpha_{k}} \dot{s}_{k} U_{s_{k-1} \cdots s_{1}} \dot{s}_{k}^{-1}$, and $U_{\tau^{\prime}} \subseteq U\left(s_{k}^{-1} w, x, s_{k} y\right)$. Then the map

$$
\lambda: x_{\alpha_{k}}\left(t_{k}\right) \dot{s}_{k} \tilde{u} \dot{s}^{-1} \rightarrow \tilde{u}
$$

is a surjective morphism defined over $k$ from $U_{\tau}$ to $U_{\tau^{\prime}}$ (as the projection from $U_{w}$ to $\dot{s}_{k} U_{s_{k-1} \cdots s_{1}} \dot{s}_{k}^{-1}$ in the factorization given above, followed by the inner automorphism by an element of $G(k)$ ). This completes our discussion of the proof of the lemma.

We now have a reduction process for cells, $U_{\tau} \rightarrow U_{\tau^{\prime}}$, as in the preceding lemma. Let $u \in U_{\tau}$ correspond to $u^{\prime} \in U_{\tau^{\prime}}$ as in the lemma. Then the structure equation $u \dot{w} u_{1} s=\dot{y} v \dot{x}^{-1}$ satisfied by $u$ corresponds to the structure equation $u^{\prime} \dot{s}_{k}^{-1} \dot{w} u_{1}^{\prime} s^{\prime}=\dot{y}^{\prime} v \dot{x}^{\prime-1}$ satisfied by $u^{\prime}$, with uniquely determined factors $\left\{u, u_{1}, s, v\right\}$ and $\left\{u^{\prime}, u_{1}^{\prime}, s^{\prime}, v^{\prime}\right\}$. The next lemma shows how the elements $u^{\prime}, u_{1}^{\prime}, v^{\prime}$ in $U$ and $s^{\prime} \in T$ are related to $u, u_{1}, v$ in $U$ and $s \in T$, using the standard form and facts about the multiplicative structure of the Chevalley group such as the decomposition $U=U_{x} U_{x w_{0}}$ for elements $x \in W$. It is also shown that the process is reversible, assuming $u \in U_{\tau}$ is known.

Lemma 3.4. Suppose that the cell $U_{\tau} \subseteq U(w, x, y)$ maps onto the cell $U_{\tau^{\prime}} \subseteq$ $U\left(s_{k-1} \cdots s_{1}, x^{\prime}, y^{\prime}\right)$ as in cases (i)-(iii) of Lemma 3.1, and let the structure equation satisfied by $u \in U_{\tau}$ be $u \dot{w} u_{1} s=\dot{y} v \dot{x}^{-1}$ with factors $u \in U_{\tau}, u_{1} \in U, s \in T$, and $v \in U_{x^{-1}}$ uniquely determined by $u \in U_{\tau}$. Let $u \rightarrow u^{\prime}=\lambda(u)$ with $u^{\prime} \in U_{\tau^{\prime}}$ as in Lemma 3.3, and consider the structure equation satisfied by $u^{\prime}$ with factors $u^{\prime} \in U, s^{\prime} \in T, u_{1}^{\prime} \in U$, and $v^{\prime} \in U_{x^{-1}}$ in each of the cases. Then the factors $u^{\prime}, u_{1}^{\prime}, s^{\prime}$ and $v^{\prime}$ are given as in the proof of the lemma. Conversely, assuming $u \in U_{\tau}$ is known, $u_{1}, s$ and $v$ are obtained from $u^{\prime}, s^{\prime}, u_{1}^{\prime}$, and $v^{\prime}$, as shown in the proof of the lemma.

In case (i), we have $\tau_{k} \tau_{k-1}^{-1}=s_{k}, \ell\left(s_{k} y\right)<\ell(y)$ and $U_{\tau^{\prime}}=\dot{s}_{k}^{-1} U_{\tau} \dot{s}_{k}$. Then the equation satisfied by $u \in U_{\tau}$ is $u \dot{w} u_{1} s=\dot{y} v \dot{x}^{-1}$ with $u=\dot{s}_{k} u^{\prime} \dot{s}_{k}^{-1}$ and $u^{\prime} \in U_{\tau^{\prime}}$. It becomes the equation for $u^{\prime} \in U_{\tau^{\prime}}$ after multiplication by $\dot{s}_{k}^{-1}$, and the lemma is proved in this case.

For the proof in case (ii) recall that $\tau_{k} \tau_{k-1}^{-1}=1$ and $\ell\left(s_{k} y\right)<\ell(y)$. Then, using the standard form for $u$, the structure equation satisfied by $u=x_{\alpha_{k}}(t) \dot{s}_{k} \tilde{u} \dot{s}_{k}^{-1} \in U_{\tau}$ is

$$
x_{\alpha_{k}}(t) \dot{s}_{k} \tilde{u} \dot{s}_{k}^{-1} \dot{w} u_{1} s=\dot{y} v \dot{x}^{-1},
$$

with $x_{\alpha_{k}}(t) \in U_{\alpha_{k}}^{*}, \tilde{u} \in U_{s_{k}^{-1} w}, s \in T, u_{1} \in U, v \in U_{x^{-1}}$. We want to derive an equation of the form

$$
u^{\prime} s_{k}^{-1} w u_{1}^{\prime} s^{\prime}=\dot{y} v^{\prime} \dot{x}^{-1}
$$

with $u^{\prime}=\pi\left(x_{\alpha_{k}}\left(-t^{-1}\right) \tilde{u}\right) \in U_{\tau^{\prime}}, s^{\prime} \in T, u_{1}^{\prime} \in U$ and $v^{\prime} \in U_{x^{-1}}$, where $\pi$ is the 
projection $U \rightarrow U_{s_{k}^{-1} w}$ as in the proof of Lemma 3.3. As

$$
\dot{s}_{k} x_{\alpha_{k}}\left(-t^{-1}\right) \dot{s}_{k}^{-1}=\dot{s}_{k}^{-1} x_{\alpha_{k}}\left(-t^{-1}\right) \dot{s}_{k},
$$

we can multiply the equation for $x_{\alpha_{k}}(t) \dot{s}_{k} \tilde{u} \dot{s}_{k}^{-1} \in U_{\tau}$ by $\dot{s}_{k} \dot{s}_{k}^{-1}$ and apply the $\mathrm{SL}_{2}-$ IDENTITY from Section 2 to obtain

$$
\dot{s_{k}} x_{\alpha_{k}}\left(-t^{-1}\right) \dot{s_{k}} h_{\alpha_{k}}(t) x_{\alpha_{k}}\left(-t^{-1}\right) \tilde{u} \dot{s}_{k}^{-1} \dot{w} u_{1} s=\dot{y} v \dot{x}^{-1} .
$$

One has $x_{\alpha_{k}}\left(-t^{-1}\right) \tilde{u}=\pi\left(x_{\alpha_{k}}\left(-t^{-1}\right) \tilde{u}\right) u^{*}$ for $u^{*} \in U_{s_{k}^{-1} w w_{0}}$ so the equation becomes $\pi\left(x_{\alpha_{k}}\left(-t^{-1}\right) \tilde{u}\right) \dot{s}_{k}^{-1} \dot{w}\left(\dot{s}_{k}^{-1} \dot{w}\right)^{-1} u^{*} \dot{s}_{k}^{-1} \dot{w} u_{1} s=\dot{y} \dot{y}^{-1}\left(\dot{s}_{k} x_{\alpha_{k}}\left(-t^{-1}\right) \dot{s}_{k}^{-1} \dot{s}_{k}^{2} h_{\alpha_{k}}(t)\right)^{-1} \dot{y} v \dot{x}^{-1}$ where $\left(\dot{s}_{k}^{-1} \dot{w}\right)^{-1} u^{*} \dot{s}_{k}^{-1} \dot{w} \in U$ because $u^{*} \in U_{s_{k}^{-1} w w_{0}}$. Note also that $\dot{s}_{k}^{2} h_{\alpha_{k}}(t)=$ $h_{\alpha_{k}}(-t)$, and that the right side of the equation is

$$
\dot{y} \dot{y}^{-1}\left(h_{\alpha_{k}}(-t)\right)^{-1} \dot{y} \dot{y}^{-1} \dot{s}_{k}\left(x_{\alpha_{k}}\left(-t^{-1}\right)^{-1}\right) \dot{s}_{k}^{-1} \dot{y} v \dot{x}^{-1} .
$$

Because $\ell\left(s_{k} y\right)<\ell(y)$, one has $\dot{y}^{-1} \dot{s}_{k} x_{\alpha_{k}}\left(-t^{-1}\right) \dot{s}_{k}^{-1} \dot{y} \in U$, and we consider first the case where $\dot{y}^{-1} \dot{s}_{k} x_{\alpha_{k}}\left(-t^{-1}\right) \dot{s}_{k}^{-1} \dot{y} \in U_{x^{-1}}$. Then the equation above becomes the structure equation for $u^{\prime} \in U_{\tau^{\prime}}$ with $u^{\prime}=\pi\left(x_{\alpha_{k}}\left(-t^{-1}\right) \tilde{u}\right), u_{1}^{\prime}=\left(\dot{s}_{k}^{-1} \dot{w}\right)^{-1} u^{*} \dot{s}_{k}^{-1} \dot{w} u_{1}$,

$$
s^{\prime}=s\left(\dot{x} \dot{y}^{-1}\left(h_{\alpha_{k}}(-t)\right)^{-1} \dot{y} \dot{x}^{-1}\right)^{-1} \text {, }
$$

and $v^{\prime}$ is

$$
\dot{y}^{-1} \dot{s}_{k}\left(x_{\alpha_{k}}\left(-t^{-1}\right)\right)^{-1} \dot{s}_{k}^{-1} \dot{y} v \in U_{x^{-1}}
$$

conjugated by $\dot{y}^{-1} h_{\alpha_{k}}(-t)^{-1} \dot{y}$. Note that $\dot{y}^{-1} h_{\alpha_{k}}(-t)^{-1} \dot{y} \in T$, and that we have conjugated this element past $\dot{y} v \dot{x}^{-1}$ and brought the result to the left-hand side as a factor of $s^{\prime}$. We have also used the fact that $U_{x^{-1}}$ is invariant under conjugation by elements of $T$.

For the reversibility, consider $u^{\prime}, u_{1}^{\prime}, s^{\prime}, v^{\prime}$ and $u=x_{\alpha_{k}}(t) \dot{s}_{k} \tilde{u} \dot{s}_{k}^{-1}$ in $U_{\tau}$. Then $x_{\alpha_{k}}\left(-t^{-1}\right) \tilde{u}=u^{\prime} u^{*}$, so $u^{*}=\left(u^{\prime}\right)^{-1}\left(x_{\alpha_{k}}\left(-t^{-1}\right) \tilde{u}\right)$. Then $s=s^{\prime}\left(\dot{x} \dot{y}^{-1} h_{\alpha_{k}}(-t) \dot{y} \dot{x}^{-1}\right)$, $u_{1}=\left(\left(\dot{s}_{k}^{-1} \dot{w}\right)^{-1} u^{*} \dot{s}_{k}^{-1} \dot{w}\right)^{-1} u_{1}^{\prime}$ and

$$
v=\left(\dot{y}^{-1} \dot{s}_{k} x_{\alpha_{k}}\left(-t^{-1}\right) \dot{s}_{k}^{-1} \dot{y}\right)^{-1} \dot{y}^{-1} h_{\alpha_{k}}(-t)^{-1} \dot{y} v^{\prime} \dot{y}^{-1} h_{\alpha_{k}}(-t) \dot{y} \in U_{x^{-1}},
$$

completing the proof of reversibility in this case, using the fact again that $U_{x^{-1}}$ is invariant under conjugation by elements of $T$.

Now we have to discuss the case $\dot{y}^{-1} \dot{s}_{k} x_{\alpha_{k}}\left(-t^{-1}\right) \dot{s}_{k}^{-1} \dot{y} \notin U_{x^{-1}}$. Then we obtain a new formula for $v^{\prime}$ as follows. We have

$$
\dot{y}^{-1} \dot{s}_{k} x_{\alpha_{k}}\left(-t^{-1}\right)^{-1} \dot{s}_{k}^{-1} \dot{y} v=v^{\prime} v^{\prime \prime},
$$

with uniquely determined factors $v^{\prime} \in U_{x^{-1}}$ and $v^{\prime \prime} \in U_{x^{-1} w_{0}}$. Then $v^{\prime \prime} \dot{x}^{-1}=$ $\dot{x}^{-1} \dot{x} v^{\prime \prime} \dot{x}^{-1}$ with $\dot{x} v^{\prime \prime} \dot{x}^{-1} \in U$. Then the structure equation in this case for $u^{\prime}=$ 
$\pi\left(x_{\alpha_{k}}\left(-t^{-1}\right) \tilde{u}\right)$ has as factors $u_{1}^{\prime}=\left(\dot{s}_{k}^{-1} \dot{w}\right)^{-1} u^{*} \dot{s}_{k}^{-1} \dot{w} u_{1} s^{\prime}\left(\dot{x} v^{\prime \prime} \dot{x}^{-1}\right)^{-1}\left(s^{\prime}\right)^{-1}, s^{\prime}$ as in the first case, and $v^{\prime}$ as defined at the beginning of this paragraph, conjugated by $\dot{y}^{-1} h_{\alpha_{k}}(-t)^{-1} \dot{y}$.

For the reversibility in this case, suppose we have $u=x_{\alpha_{k}}(t) \dot{s}_{k} \tilde{u} \dot{s}_{k}^{-1} \in U_{\tau}$, $u^{\prime}=\pi\left(x_{\alpha_{k}}\left(-t^{-1}\right) \tilde{u}\right)$, and $u_{1}^{\prime}, s^{\prime}$, and $v^{\prime}$ as above. We have to solve for $u_{1}, s$, and $v$. Then, after reversing the conjugation by $\dot{y}^{-1} h_{\alpha_{k}}(-t)^{-1} \dot{y}$, we obtain

$$
\left(\dot{y}^{-1} \dot{s}_{k} x_{\alpha_{k}}\left(-t^{-1}\right) \dot{s}_{k}^{-1} \dot{y}\right)^{-1} v^{\prime}=v\left(v^{\prime \prime}\right)^{-1} .
$$

Then $v$ is the projection of the left-hand side in $U_{x^{-1}}$. Then from

$$
u_{1}^{\prime}=\left(\dot{s}_{k}^{-1} \dot{w}\right)^{-1} u^{*} \dot{s}_{k}^{-1} \dot{w} u_{1} s^{\prime}\left(\dot{x} v^{\prime \prime} \dot{x}^{-1}\right)^{-1}\left(s^{\prime}\right)^{-1},
$$

we can express $u^{*}$ as the projection of $x_{\alpha_{k}}\left(-t^{-1}\right) \tilde{u}$ in $U_{s_{k}^{-1} w w_{0}}$ and $\left(\dot{x} v^{\prime \prime} \dot{x}^{-1}\right)^{-1}$ from $x_{\alpha_{k}}\left(-t^{-1}\right)$ and $v^{\prime}$, so we recover $u_{1}$. Finally the element $s$ is computed as in the first case. Therefore we have obtained $u_{1}, s$, and $v$, completing the proof of reversibility in this case.

In case (iii), the structure equation satisfied by $u=x_{\alpha_{k}}(t) \dot{s}_{k} \tilde{u} \dot{s}_{k}^{-1} \in U_{\tau}$ is

$$
x_{\alpha_{k}}(t) \dot{s}_{k} \tilde{u} \dot{s}_{k}^{-1} \dot{w} u_{1} s=\dot{y} v \dot{x}^{-1}
$$

as in case (ii). This time $\ell\left(s_{k} y\right)>\ell(y)$, so $\dot{y}^{-1} x_{\alpha_{k}}(t) \dot{y} \in U$ and the structure equation for $\tilde{u} \in U_{\tau^{\prime}}$ becomes

$$
\tilde{u} \dot{s}_{k}^{-1} \dot{w} u_{1} s=\dot{s}_{k}^{-1} \dot{y} \dot{y}^{-1} x_{\alpha_{k}}(t)^{-1} \dot{y} v \dot{x}^{-1},
$$

and the rest of the proof is handled as in case (ii), depending on whether $\dot{y}^{-1} x_{\alpha_{k}}(t) \dot{y} \in$ $U_{x^{-1}}$ or not. This completes the proof of the lemma.

\section{Solution of the structure equation}

Let $\ell, m, n \in N^{*}$ be representatives of $w, x, y$ in $W$, and let $w=s_{k} \cdots s_{1}$ be a fixed reduced expression of $w$. From the Introduction, the structure constants of standard basis elements $c_{\ell}, c_{m}, c_{n}$ of the Hecke algebra $H$ of an induced representation $\gamma=\psi^{G}$ of $G$ are given by the formula

$$
\left[c_{\ell} c_{m}: c_{n}\right]=\sum_{u \ell u_{1}=n v m^{-1} \in U \ell U \cap n U_{m^{-1}} m^{-1}} \psi\left(\left(u u_{1}\right)^{-1} v\right),
$$

where $U \ell U \cap n U_{m^{-1}} m^{-1}$ is the set of elements $u \in U, u_{1} \in U, v \in U_{m^{-1}}$ satisfying the equation

$$
u \ell u_{1}=n v m^{-1} \text {. }
$$

The elements $\ell, m, n$ are multiples of $\dot{w}, \dot{x}, \dot{y}$ by elements of $T, \ell=\dot{w} s, m=\dot{x} s^{\prime}$, etc. for elements $s, s^{\prime}, s^{\prime \prime}$ in $T$. The equations $u \ell u_{1}=n v m^{-1}$ as above can be 
rewritten in the form $u \dot{w} \hat{u}_{1} \hat{s}=\dot{y} \hat{v} \dot{x}^{-1}$ for $u \in U, \hat{s}=s\left(\dot{x} s^{\prime \prime}\left(s^{\prime}\right)^{-1} \dot{x}^{-1}\right)^{-1} \in T, \hat{u}_{1}=$ $s u_{1} s^{-1} \in U, \hat{v}=s^{\prime \prime} v\left(s^{\prime \prime}\right)^{-1} \in U_{x^{-1}}$, using the fact that the subsets $U_{x}$, for $x \in W$, are invariant under conjugation by elements of $T$. Each element $u \in U$ satisfying the equation above belongs to the set $U(w, x, y)$, and consequently $u \in U_{\tau}$ for a cell $U_{\tau}$ defined by a $K$-sequence $\tau$ for the elements $\{w, x, y\}$ in $W$. An algorithm for the solutions $u, \hat{u}_{1}, \hat{v}$ of these equations is the main result of this section.

As $u \in U_{\tau}$ is known in terms of the root subgroups from the main result of [Curtis 1988], the problem is to calculate $\hat{u}_{1}$ and $\hat{v}$ in terms of a given expression of $u$.

The following remarks may throw some light on these problems. Let $\tau$ be a $K$-sequence for $w, x, y$ in $W$, and let $U_{\tau}$ be the corresponding cell in $U(w, x, y)$. Each element $u \in U_{\tau}$ satisfies a structure equation

$$
u \dot{w} b=\dot{y} v \dot{x}^{-1}
$$

with $b \in B$ and $v \in U_{x^{-1}}$. It is known that the elements $b$ and $v$ in the structure equation are uniquely determined by $u$ [Curtis 2009, Lemma 2.4]. A main theorem in [Curtis 2009] was an inductive construction of the solutions of the structure equation. Theorem 4.1 below gives more information, and in a sense, calculates $b$ and $v$ from an expression of $u$ in standard form using a fixed reduced expression of $w$. In particular, this result determines, for each element $u \in U_{\tau}$, the solutions $\left(u_{1}, v\right)$, of the equations $u \ell u_{1}=n v m^{-1}$ with $u \in U_{\tau}, u_{1} \in U$, and $v \in U_{x^{-1}}$, needed for the structure constants of $H$.

Theorem 4.1. Let $w, x, y$ be elements of $W$, and let $U_{\tau}$ be a cell associated with a $K$-sequence $\tau$ for $w, x, y$, and a fixed reduced expression $w=s_{k} \cdots s_{1}$ for $w$. The algorithm given below determines the set of elements $s \in T, u_{1} \in U$ and $v \in U_{x^{-1}}$ satisfying the equation $u \dot{w} u_{1} s=\dot{y} v \dot{x}^{-1}$, for a given element $u \in U_{\tau}$. The possibility that the set of solutions is empty is not excluded.

Let $w, x, y$, the cell $U_{\tau}$, and $w=s_{k} \cdots s_{1}$ be as in the hypothesis of the theorem. Let $u$ be a fixed element of $U_{\tau}$. With these as a starting point, the algorithm gives the elements $s \in T, u_{1} \in U$ and $v \in U_{x^{-1}}$ satisfying the equation stated in the theorem. It is proved by induction on $\ell(w)$.

We begin with the case $\ell(w)=1$, so $\dot{w}=\dot{s}_{1}$ and let $\tau=\left(\tau_{1}, \tau_{0}\right)$ be a $K$-sequence for $\left(s_{1}, x, y\right)$ corresponding to one of the three cases in Lemma 3.1.

Case (i). $\tau_{1}=s_{1}, \ell\left(s_{1} y\right)<\ell(y)$. Then $U_{\tau_{0}}=1, U_{\tau}=\dot{s}_{1} U_{\tau_{0}} \dot{s}_{1}^{-1}=1, s_{1} x=y$ and it is easily proved using Lemma 83 of [Steinberg 1968] that $\dot{s}_{1} \dot{x}=\dot{y}$. Then there is a unique solution, namely $(1,1,1)$, of the structure equation $u \dot{s}_{1} b=\dot{s}_{1} \dot{x} v(\dot{x})^{-1}$.

Case (ii). $\tau_{1}=\tau_{0}$, and $\ell\left(s_{1} y\right)<\ell(y)$. In this case the definition of $K$-sequence implies $x=y$. We also have (by part (ii) of the proof of Lemma 3.3) $U_{\tau}=U_{\alpha_{1}}^{*}$. First assume $\ell(x)=1$. Then the assumptions imply that $x=s_{1}, \dot{x}=\dot{y}=\dot{s}_{1}$, and for each element $u \in U_{\alpha_{1}}^{*}$ there is a unique solution of the structure equation $u \dot{s}_{1} b=\dot{s}_{1} v \dot{s}_{1}^{-1}$ 
by the $\mathrm{SL}_{2}$-IDENTITY, so that quadruples $\left(u, s, u_{1}, v\right)$ with $u \in U_{\tau}, b=u_{1} s \in B$, and $v \in U_{x^{-1}}$ satisfying the equation exist, and are known. Now let $\ell(x)>1$; then $\ell\left(s_{1} x\right)<\ell(x)$ implies $\dot{x}=\dot{s}_{1} \dot{x}_{1}$ with $\ell\left(s_{1} x_{1}\right)>\ell\left(x_{1}\right)$. For each $v \in U_{\alpha_{1}}^{*}$ one has $\dot{x}_{1}^{-1} v \dot{x}_{1} \in U$ because $\ell\left(s_{1} x_{1}\right)>\ell\left(x_{1}\right)$. Moreover

$$
\dot{s}_{1} \dot{x}_{1}\left(\dot{x}_{1}^{-1} v \dot{x}_{1}\right) \dot{x}_{1}^{-1} \dot{s}_{1}^{-1}=\dot{s}_{1} v \dot{s}_{1}^{-1} \in U_{-}
$$

so $\dot{x}_{1}^{-1} v \dot{x}_{1} \in U_{x^{-1}}$. The unique solution of the structure equation $u \dot{s}_{1} b=\dot{s}_{1} v \dot{s}_{1}^{-1}$ with $u \in U_{\alpha_{1}}^{*}$ from the case $\ell(x)=1$ now yields the unique solution $\left(u, b, \dot{x}_{1}^{-1} v \dot{x}_{1}\right)$ of the structure equation for $\left(s_{1}, x, y\right)$, namely

$$
u \dot{s}_{1} b=\dot{y} \dot{x}_{1}^{-1} v \dot{x}_{1} \dot{x}^{-1}
$$

as $\dot{y} \dot{x}_{1}^{-1}=\dot{s}_{1}$ and $\dot{x}_{1} \dot{x}^{-1}=\dot{s}_{1}^{-1}$. Note that in case (ii) there is no solution of the structure equation $u \dot{s}_{1} b=\dot{y} v \dot{x}^{-1}$ in case $u=1$ and $x=y$ as this would contradict the fact that $B \dot{y}^{-1} \dot{s}_{1} B \neq B \dot{x}^{-1} B$ by the uniqueness part of the Bruhat decomposition.

Case (iii). $\tau_{1}=s_{1}$, and $\ell\left(s_{1} y\right)>\ell(y)$. In this case $U_{\tau}=U_{\alpha_{1}}$ and the unique solution of the structure equation $u \dot{s}_{1} b=\dot{y} v \dot{x}^{-1}$ for $u \in U_{\alpha_{1}}$ is

$$
\left(u, h_{\alpha_{1}}(-1), \dot{y}^{-1} u \dot{y}\right),
$$

for each $u \in U_{\alpha_{1}}$, noting that $\dot{y}^{-1} u \dot{y} \in U_{x^{-1}}$, and $\dot{y} \dot{x}^{-1}=\dot{s}_{1}^{-1}=\dot{s}_{1} h_{\alpha_{1}}(-1)$ by [Steinberg 1968, Lemma 83] again. This completes the discussion of the solutions of the structure equation for the case $\ell(w)=1$.

We now proceed to the general case, with $\ell(w)>1$. Let $\tau$ be a $K$-sequence for $w, x, y$ and let $u \in U_{\tau}$. Let $u$ correspond to $u^{\prime}=\lambda(u) \in U_{\tau^{\prime}}$ (as in Lemma 3.3) for the $K$-sequence $\tau^{\prime}$ for $s_{k-1} \ldots s_{1}, x^{\prime}, y^{\prime}$ in one of the three cases of Lemma 3.1, and let

$$
u^{\prime} s_{k-1} \ldots s_{1} u_{1}^{\prime} s^{\prime}=\dot{y}^{\prime} v^{\prime} \dot{x}^{\prime}
$$

be the structure equation satisfied by $u^{\prime}$. By the induction hypothesis, the factors $u_{1}^{\prime}, s^{\prime}$, and $v^{\prime}$ of the structure equation for $u^{\prime}$ are determined by $u^{\prime}$. As $u^{\prime}=\lambda(u)$, the elements $u_{1}, s$, and $v$ satisfying the equation $u \dot{w} b=u \dot{w} u_{1} s=\dot{y} v \dot{x}^{-1}$ are determined by $u$, using Lemma 3.4. This completes the proof of the theorem.

At the beginning of the section, it was explained how the solutions $u \in U, u_{1} \in U$, and $v \in U_{x^{-1}}$ of the equations $u \ell u_{1}=n v m^{-1}$, for $\ell, m, n \in N^{*}$, required for the formulas for the structure constants are obtained from the solutions $u \in U_{\tau}, u_{1} \in U$, $s \in T, v \in U_{x^{-1}}$ of the equations $u \dot{w} u_{1} s=\dot{y} v \dot{x}^{-1}$ solved by the algorithm in Theorem 4.1. For this step, it is necessary to determine the elements $t, t^{\prime}, t^{\prime \prime}$ in $T$ such that $\ell=\dot{w} t, m=\dot{x} t^{\prime}, n=\dot{y} t^{\prime \prime}$, in order to transform the first set of equations to the second by the algorithms for multiplication the Chevalley group. This information can be obtained from Steinberg's proof of Theorem 49 in [Steinberg $1968, \S 14]$, in case $\psi^{G}$ is a Gelfand-Graev representation. The theorem states that 
the Hecke algebra $H$ of a Gelfand-Graev representation is a commutative algebra, and the proof is obtained by constructing a certain antiautomorphism $f$ of the Chevalley group $G$ whose extension to the group algebra is at the same time an antiautomorphism of the group algebra and whose restriction to the Hecke algebra $H$ is the identity. As shown in [Steinberg 1968], the representatives $\ell \in N^{*}$ of the basis elements of $H$ have the form $t \dot{w}$ for elements $w \in W$ such that $w=w_{0} w_{\pi}$, where $w_{0}$ is the element of maximal length in $W$ and $w_{\pi}$ is the element of maximal length in the subgroup of the Weyl group generated by the reflections taken from a subset $\pi$ of the set of simple roots, and $t$ is an element of $T$ such that $t \dot{w}$ is fixed by the antiautomorphism $f$. From the discussion on page 262 of [Steinberg 1968], it follows directly that $\dot{w} t$, with $w=w_{0} w_{\pi}$ as above, represents a basis element of $H$, fixed by the antiautomorphism $f$, whenever $t$ commutes with $w_{\pi}$.

We recall the connection between the solutions of the equation $u \ell u_{1}=n v m^{-1}$ and the solutions of the equation $u \dot{w} \hat{u}_{1} \hat{s}=\dot{y} \hat{v} \dot{x}^{-1}$ with $\ell=\dot{w} s, m=\dot{x} s^{\prime}, n=\dot{y} s^{\prime \prime}$, $s, s^{\prime}, s^{\prime \prime} \in T$ and $\hat{u}_{1}=s u_{1} s^{-1} \in U, \hat{v}=s^{\prime \prime} v\left(s^{\prime \prime}\right)^{-1} \in U_{x^{-1}}$, for $u \in U$. For a solution $u \in U$, we have $u \in U(w, x, y)$ so $u \in U_{\tau}$ for a $K$-sequence $\tau$ for $w, x, y$. We can now state a formula for the structure constants $\left[c_{\ell} c_{m}: c_{n}\right]$ based on Theorem 4.1.

Corollary 4.2. The structure constants are given by the formula

$$
\left[c_{\ell} c_{m}: c_{n}\right]=\sum_{\tau} \sum_{u \in U_{\tau}} \psi\left(\left(u u_{1}\right)^{-1} v\right)
$$

where for each $K$-sequence $\tau$ for $w, x, y$, the sum is taken over solutions of the equation $u \dot{w} \hat{u}_{1} \hat{s}=\dot{y} \hat{v} \dot{x}^{-1}$ obtained by Theorem 4.1, with $u \in U_{\tau}$ and $\hat{u}_{1}, \hat{v}, \hat{s}$ satisfying the conditions $\hat{u}_{1}=s u_{1} s^{-1} \in U, \hat{v}=s^{\prime \prime} v\left(s^{\prime \prime}\right)^{-1} \in U_{x^{-1}}$ and $\hat{s}=s\left(\dot{x} s^{\prime \prime}\left(s^{\prime}\right)^{-1} \dot{x}^{-1}\right)^{-1} \in T$. If there are no solutions satisfying these conditions, then the structure constant is zero.

We end this section with two problems for further research.

1. The first problem is to apply the algorithm obtained in Theorem 4.1 and Corollary 4.2 to obtain formulas for the structure constants $\left[c_{\ell} c_{m}: c_{n}\right]$ which can be used to give a combinatorial proof of the existence of the homomorphisms $f_{T}$ mentioned in the Introduction.

2. The second problem is to develop a theory of cells for Chevalley groups over a $p$-adic field $K$, using the Bruhat decomposition for these groups obtained by Iwahori and Matsumoto [1965].

\section{Example: application to $\mathrm{SL}_{2}(k)$}

Let $G$ be the Chevalley group $\mathrm{SL}_{2}(k)$ for a finite field $k$ of odd characteristic, and let $H$ be the Hecke algebra of a Gelfand-Graev representation of $G$. Gelfand and 
Graev [1962a] stated formulas for the structure constants of the standard basis of $H$, and calculated the irreducible representations of $H$.

As an application of the ideas in Section 4, we shall calculate the structure constants of $H$ relative to the standard basis of the Hecke algebra, and apply them to give a self-contained proof, different from the one obtained by Gelfand and Graev, of formulas for the irreducible representations of $H$ (for another approach, using the Deligne-Lusztig character formula, see [Curtis 1993, §5]).

We begin with a Gelfand-Graev character $\psi^{G}$ of $G=\mathrm{SL}_{2}(k)$, for a linear character $\psi$ of $U$ in general position. Then we may assume that

$$
\psi\left(\begin{array}{ll}
1 & \alpha \\
0 & 1
\end{array}\right)=\chi(\alpha), \quad\left(\begin{array}{ll}
1 & \alpha \\
0 & 1
\end{array}\right) \in U, \quad \alpha \in k
$$

for a nontrivial additive character $\chi$ on $k$. The standard basis elements of the Hecke algebra $H$ of $\psi^{G}$ are the elements

$$
c_{\lambda}=q e_{\psi} n_{\lambda} e_{\psi}, \quad n_{\lambda}=\left(\begin{array}{cc}
0 & \lambda \\
-\lambda^{-1} & 0
\end{array}\right), \quad \lambda \neq 0, \quad q=|k|
$$

together with the identity element $e_{\psi}$ and one other basis element $e_{-1}=e_{\psi}\left(\begin{array}{rr}-1 & 0 \\ 0 & -1\end{array}\right) e_{\psi}$, where

$$
e_{\psi}=|U|^{-1} \sum_{u \in U} \psi\left(u^{-1}\right) u
$$

as in the Introduction.

Lemma 5.1. The algebra $H$ is commutative with identity element $e_{\psi}$. One has $e_{-1}^{2}=e_{\psi}$, and $e_{-1} c_{\lambda}=c_{-\lambda}$ for each $\lambda \neq 0$. The other nonzero structure constants of $H$ for the standard basis elements are as follows. For $c_{\lambda}, c_{\mu}, c_{\nu}$ as above one has

$$
\left[c_{\lambda} c_{\mu}: c_{\nu}\right]=\chi\left(\lambda \mu \nu^{-1}+\lambda \mu^{-1} v+\lambda^{-1} \mu \nu\right)
$$

and

$$
\left[c_{\lambda} c_{\lambda}: e_{-1}\right]=q, \quad\left[c_{\lambda} c_{-\lambda}: e_{\psi}\right]=q,
$$

for $\lambda, \mu, v \neq 0$ in $k$, and $q=|k|$.

The structure constants are computed using the formula at the beginning of $\S 4$ and the solutions of the structure equation

$$
u \ell u_{1}=n v(m)^{-1}
$$

with $\ell, m, n \in N$ (see [Curtis 2009, §3] for more details).

The group $G=\mathrm{SL}_{2}(k)$ can be viewed as the group of fixed points by the usual Frobenius endomorphism $F$ of the semisimple algebraic group $\mathrm{SL}_{2}(\bar{k})$, over the algebraic closure $\bar{k}$ of $k$. There are two conjugacy classes of $F$-stable maximal tori in $\mathrm{SL}_{2}(\bar{k})$ with representatives in the finite group $G$ given by the split torus 
$T_{0}$ consisting of the matrices $\left(\begin{array}{cc}\mu & 0 \\ 0 & \mu^{-1}\end{array}\right)$ with $\mu \neq 0$ in $k$, and the Coxeter torus $T_{1}$, isomorphic to the set $C$ of elements $\xi$ of norm 1 in the quadratic extension of $k$, that is, $\xi^{q+1}=1$. The main theorem on the representations of the Hecke algebra $H$ of a Gelfand-Graev representation of $G$ states that the irreducible representations of $H$ factor through the group algebra of one of the maximal tori of $G$. More precisely, one has:

Theorem 5.2. Each irreducible representation $f$ of the Hecke algebra $H$ of a Gelfand-Graev representation of $G$ can be factored as

$$
f=\theta \circ f_{T},
$$

where $f_{T}$ is a homomorphism, independent of $\theta$, of $H$ into the group algebra of a maximal torus $T$ of $G$, and $\theta$ is an irreducible representation of the group algebra of the maximal torus. The homomorphisms from $H$ into the group algebras of the two types of maximal tori are given as follows. For the split torus $T_{0}$, consisting of diagonal matrices with entries in $k^{*}$, the homomorphism $f_{T_{0}}: H \rightarrow \mathbb{C} T_{0}$ is given by

$$
f_{T_{0}}\left(c_{\lambda}\right)=\sum_{t} \chi\left(\lambda\left(t+t^{-1}\right)\right)\left(\begin{array}{cc}
t & 0 \\
0 & t^{-1}
\end{array}\right), \quad t \in k^{*}, \quad \text { and } \quad f_{T_{0}}\left(e_{-1}\right)=\left(\begin{array}{rr}
-1 & 0 \\
0 & -1
\end{array}\right),
$$

where $c_{\lambda}$ is a standard basis element of $H$ as above. For the Coxeter torus, the homomorphism $f_{T_{1}}: H \rightarrow \mathbb{C} C$ is given by

$$
f_{T_{1}}\left(c_{\lambda}\right)(\xi)=-\chi\left(\lambda\left(\xi+\xi^{-1}\right)\right), \quad \xi \in C, \quad \text { and } \quad f_{T_{1}}\left(e_{-1}\right)=\xi_{-1},
$$

where $\xi_{-1}$ is the unique element in $C$ of order two.

Lemma 5.3. Let $a, b \in k$. Then:

$$
\begin{gathered}
\sum_{t \in k^{*}} \chi(a t)=-1+q \delta_{a, 0} . \\
\sum_{t \in k^{*}} \chi\left(a t+b t^{-1}\right)=\sum_{t \in k^{*}} \chi\left(t+a b t^{-1}\right)+q \delta_{a, 0} \delta_{b, 0} .
\end{gathered}
$$

(iii) For the Coxeter torus $C$, we first note that $\xi+\xi^{-1} \in k$ because $\xi^{q+1}=1$ for $\xi \in C$ implies that $\xi+\xi^{-1}=\xi+\xi^{q} \in k$. Let $\xi \in C, \eta \in F_{q^{2}}$. Then

$$
\sum_{\xi \in C} \chi\left(\xi \eta+\xi^{q} \eta^{q}\right)=-\sum_{t \in k^{*}} \chi\left(t+\eta \eta^{q} t^{-1}\right)+\delta_{\eta, 0} q .
$$

We refer to [Chang 1976, Lemma 1.2]. Part (iii) is proved using an analysis of quadratic equations over $k$. The result, and extensions of it to $F$-stable maximal tori in general finite reductive groups, are suggested by the Davenport-Hasse Theorem on Gauss sums. 
For the proof that $f_{T_{0}}$ is a homomorphism, let $c_{\lambda}, c_{\mu}, c_{\nu}$ be standard basis elements of $H$ as above. Then

$$
c_{\lambda} c_{\mu}=\sum_{\nu}\left[c_{\lambda} c_{\mu}: c_{\nu}\right] c_{\nu}+\delta_{\lambda, \mu} q e_{-1}+\delta_{\lambda,-\mu} q e_{\psi},
$$

with the structure constants as in Lemma 5.1. We have

$$
\begin{aligned}
f_{T_{0}}\left(c_{\lambda}\right) f_{T_{0}}\left(c_{\mu}\right) & =\sum_{t \in k^{*}} \sum_{s \in k^{*}} \chi\left(\lambda\left(t s+t^{-1} s^{-1}\right)\right) \chi\left(\mu\left(s+s^{-1}\right)\right)\left(\begin{array}{cc}
t & 0 \\
0 & t^{-1}
\end{array}\right) \\
& =\sum_{t} \sum_{s} \chi\left((\lambda t+\mu) s+\left(\lambda t^{-1}+\mu\right) s^{-1}\right)\left(\begin{array}{cc}
t & 0 \\
0 & t^{-1}
\end{array}\right) \\
& =\sum_{t} \sum_{s^{\prime}} \chi\left(s^{\prime}+\left(\lambda^{2}+\mu^{2}+\lambda \mu\left(t+t^{-1}\right)\left(s^{\prime}\right)^{-1}\right)\right)\left(\begin{array}{cc}
t & 0 \\
0 & t^{-1}
\end{array}\right)
\end{aligned}
$$

by Lemma 5.3(ii), and have to show this is equal to

$$
\begin{aligned}
\sum_{t} \sum_{\nu}\left[c_{\lambda} c_{\mu}: c_{\nu}\right] & f_{T_{0}}\left(c_{\nu}\right)(t)\left(\begin{array}{cc}
t & 0 \\
0 & t^{-1}
\end{array}\right)+\delta_{\lambda, \mu} q\left(\begin{array}{rr}
-1 & 0 \\
0 & -1
\end{array}\right)+\delta_{\lambda,-\mu} q\left(\begin{array}{ll}
1 & 0 \\
0 & 1
\end{array}\right) \\
& =\sum_{t} \sum_{\nu} \chi\left(\lambda \mu \nu^{-1}+\lambda \mu^{-1} v+\lambda^{-1} \mu \nu+v\left(t+t^{-1}\right)\right)\left(\begin{array}{ll}
t & 0 \\
0 & t^{-1}
\end{array}\right),
\end{aligned}
$$

etc. The result is clear, by another application of Lemma 5.3(ii), in case $\lambda \neq \pm \mu$.

Now let $\lambda=\mu$. The expressions to be checked agree except possibly at $\left(\begin{array}{rr}-1 & 0 \\ 0 & -1\end{array}\right)$. At $\left(\begin{array}{rr}-1 & 0 \\ 0 & -1\end{array}\right)$, the first expression becomes

$$
\sum_{s} \chi\left(\lambda(t+1) s+\lambda\left(t^{-1}+1\right) s^{-1}\right)
$$

with $t=-1$, which is $q-1$ by Lemma 5.3. The second expression at $\left(\begin{array}{rr}-1 & 0 \\ 0 & -1\end{array}\right)$ becomes

$$
\sum_{v} \chi\left(v+\left(2 \lambda^{2}+\left(-2 \lambda^{2}\right)\right) v^{-1}\right)+q=-1+q
$$

by Lemma 5.3 again, completing the proof in this case. The proof in case $\lambda=-\mu$ is similar and will be omitted.

For the homomorphism from $H$ into the group algebra of $C$, we first have

$$
\begin{aligned}
f_{T_{1}}\left(c_{\lambda}\right) f_{T_{1}}\left(c_{\mu}\right) & =\sum_{\xi \in C} \sum_{\eta \in C} \chi\left(\lambda\left(\xi \eta+(\xi \eta)^{-1}\right)+\mu\left(\eta+\eta^{-1}\right)\right) \xi \\
& =\sum_{\xi} \sum_{\eta} \chi\left((\lambda \xi+\mu) \eta+\left(\lambda \xi^{-1}+\mu\right) \eta^{-1}\right) \xi \\
& =-\sum_{\xi} \sum_{t \in k^{*}} \chi\left(t+(\lambda \xi+\mu)\left(\lambda \xi^{q}+\mu\right) t^{-1}\right) \xi
\end{aligned}
$$


by Lemma 5.3(ii). We have to show that this is equal to

$$
\begin{aligned}
& \left.\sum_{\xi \in C} \sum_{v \in k^{*}} \chi\left(\lambda \mu v^{-1}+\lambda \mu^{-1} v+\lambda^{-1} \mu \nu\right) f_{T_{1}}\left(c_{\nu}\right)\right) \xi+\delta_{\lambda, \mu} q f_{T_{1}}\left(e_{-1}\right)+\delta_{\lambda,-\mu} q f_{T_{1}}\left(e_{\psi}\right) \\
= & -\sum_{\xi \in C} \sum_{v \in k^{*}} \chi\left(v\left(\xi+\xi^{-1}+\lambda \mu^{-1}+\lambda^{-1} \mu\right)+v^{-1} \lambda \mu\right) \xi+\delta_{\lambda, \mu} q f_{T_{1}}\left(e_{-1}\right)+\delta_{\lambda,-\mu} q f_{T_{1}}\left(e_{\psi}\right) \\
= & -\sum_{\xi} \sum_{\nu^{\prime}}\left(\chi\left(v^{\prime}+v^{\prime-1}\left(\xi+\xi^{q}\right) \lambda \mu+\lambda^{2}+\mu^{2}\right)\right) \xi+\delta_{\lambda, \mu} q f_{T_{1}}\left(e_{-1}\right)+\delta_{\lambda,-\mu} q f_{T_{1}}\left(e_{\psi}\right),
\end{aligned}
$$

where we have used Lemma 5.3(ii) and Lemma 5.1 for the structure constant formula. Together, these formulas prove the multiplication formula in case $\lambda \neq \pm \mu$.

In case $\lambda=\mu$, it is only necessary to check the expressions at $\xi=\xi_{-1}$, where $\xi_{-1}$ is the unique element of $C$ such that $\xi_{-1}^{2}=1, \xi_{-1} \neq 1$, so $\xi_{-1}=-1$ in $F_{q^{2}}$. The contribution from the first expression is

$$
\sum_{\eta} \chi\left(\lambda\left(\xi_{-1}+1\right) \eta+\lambda\left(\xi_{-1}^{-1}+1\right) \eta^{-1}\right)=-\sum_{t \in k^{*}} \chi\left(t+\left(2 \lambda^{2}+\lambda^{2}\left(\xi_{-1}+\xi_{-1}^{q}\right)\right) t^{-1}\right)+q
$$

by Lemma 5.3(iii). As $\xi_{-1}+\xi_{-1}^{q}=-2$ in $F_{q^{2}}$, this expression is equal to $1+q$ by Lemma 5.3. For the second expression at $\xi_{-1}$ we obtain

$$
-\sum_{v^{\prime}} \chi\left(v^{\prime}+\left(v^{\prime}\right)^{-1}\left(\left(\xi_{-1}+\xi_{-1}^{q}\right) \lambda^{2}+2 \lambda^{2}\right)\right)+q=q+1,
$$

completing the proof in this case.

For the remaining case $\lambda=-\mu$ it is only necessary to check both expressions at $\xi=1$ and this is immediate.

Corollary 5.4. The formulas for the irreducible representations of the Hecke algebra $\mathrm{H}$ are

$$
f\left(c_{\lambda}\right)=\theta \circ f_{T}\left(c_{\lambda}\right)=\sum_{t \in T} \chi\left(\lambda\left(t+t^{-1}\right)\right) \theta(t), \quad f\left(e_{-1}\right)=\theta(-1)
$$

for the split torus $T$, and an irreducible representation $\theta$ of $T$, and

$$
f\left(c_{\lambda}\right)=\pi \circ f_{T_{1}}\left(c_{\lambda}\right)=-\sum_{\xi \in C} \chi\left(\lambda\left(\xi+\xi^{-1}\right)\right) \pi(\xi), \quad f\left(e_{-1}\right)=\pi(-1)
$$

for the Coxeter torus $T_{1}$ represented by $C$, and an irreducible representation $\pi$ of $C$.

The fact that all the irreducible representations of $H$ are obtained in this way follows by a counting argument.

Gelfand and Graev [1962a] obtained these formulas, and pointed out that they are similar to the integral formulas for Bessel functions over $\mathbb{C}$ (see [Whittaker and Watson 1927, Chapter XVII]). They mentioned that the formulas in Corollary 5.4 can be called Bessel functions over finite fields. 
The group $G=\mathrm{SL}_{2}(k)$, for $q=|k|$ odd, has two classes of Gelfand-Graev representations. For a determination of the irreducible characters of $G$, and how they appear in the Gelfand-Graev representations, including for example the subtle cases of those of degree $\frac{1}{2}(q+1)$ and $\frac{1}{2}(q-1)$, see [Gelfand and Graev 1962a, §4] and [Digne and Michel 1991, §15.9].

\section{Example: the homomorphisms $f_{T}$ associated with principal series representations of finite Chevalley groups}

We return to the set-up described in the Introduction, with $G$ a Chevalley group over a finite field $k$, with a Borel subgroup $B=U T$ containing the torus $T$, and Weyl group $W$. Let $\psi^{G}$ be a Gelfand-Graev representation of $G$, and $H=e \mathbb{C} G e$ the Hecke algebra associated with it, with

$$
e=|U|^{-1} \sum_{u \in U} \psi\left(u^{-1}\right) u
$$

(remember that $H$ is a commutative algebra!). In this section, we give a character theoretic construction of a homomorphism $f_{T}: H \rightarrow \mathbb{C} T$ and the resulting irreducible representations of $H$. An open problem is to find a combinatorial construction of homomorphisms $f_{T}$ for twisted tori $T$. Such a result would define a family of functions associated with the Hecke algebra $H$ and maximal tori in $G$, starting from the Bessel functions over $k$ in the case of $\mathrm{SL}_{2}(k)$ and the Coxeter torus $C$. A proof would require information about the structure constants, and extensions of Lemma 5.3(iii), which would be of independent interest. Homomorphisms $f_{T}: H \rightarrow \mathbb{C} T$ from a Gelfand-Graev Hecke algebra $H$ to the group algebra of a maximal torus are known to exist, for a connected reductive algebraic group $G$ defined over a finite field, with Frobenius endomorphism $F$ ([Curtis 1993] and [Bonnafé and Kessar 2008]), and are derived using the trace formula in $\ell$-adic cohomology.

We are concerned with the principal series representations of $G$. These are the induced representations $\lambda^{G}$, where $\lambda$ is a linear character of the Borel subgroup $B$ with $U$ in its kernel, and the irreducible representations of $G$ which occur as constituents of $\lambda^{G}$ for some choice of lambda. We require the following result of Kilmoyer [1978, Proposition 6.1].

Lemma 6.1. Let $\psi^{G}$ be a fixed Gelfand-Graev character of G. Each induced character $\lambda^{G}$, as above, contains a unique irreducible constituent $\xi_{\lambda}$ which appears with multiplicity one in both $\lambda^{G}$ and the Gelfand-Graev character $\psi^{G}$.

As in [Curtis 1993], we introduce the notation $\boldsymbol{a}$ for the element of the group algebra $\mathbb{C} G$ given by

$$
\boldsymbol{a}=\sum_{g \in G} \alpha\left(g^{-1}\right) g
$$


for a complex valued function $\alpha$ on $G$. If $\alpha$ is an irreducible character of $G$, then $\boldsymbol{a}$ is a multiple of the central primitive idempotent in the group algebra associated with $\alpha$.

Theorem 6.2. Let $\boldsymbol{l}_{\lambda}$ and $\boldsymbol{x}_{\lambda}$ be the elements of the group algebra corresponding to the induced character $\lambda^{G}$ and the irreducible character $\xi_{\lambda}$ as in Lemma 6.1. Then

$$
e \boldsymbol{l}_{\lambda}=e \boldsymbol{x}_{\lambda} \neq 0
$$

and affords an irreducible representation $f_{\xi_{\lambda}}: H \rightarrow \mathbb{C}^{*}$ of $H$ of degree one, such that

$$
h\left(e \boldsymbol{x}_{\lambda}\right)=f_{\xi_{\lambda}}(h) e \boldsymbol{x}_{\lambda}, \quad h \in H .
$$

The representation $f_{\xi_{\lambda}}$ is the restriction to $H$ of the unique irreducible character of the group algebra $\mathbb{C} G$ obtained from the character $\xi_{\lambda}$ of $G$ as in Lemma 6.1.

By Lemma 6.1, the class function $\boldsymbol{l}_{\lambda}=\boldsymbol{x}_{\lambda}+\boldsymbol{y}$ where $\boldsymbol{y}$ is a linear combination of central primitive idempotents corresponding to irreducible characters of $G$ which do not appear in the Gelfand-Graev representation $\psi^{G}$. Then $e \boldsymbol{y}=0$, so $e \boldsymbol{l}_{\lambda}=e \boldsymbol{x}_{\lambda} \neq 0$ and $e \boldsymbol{x}_{\lambda}$ is a nonzero multiple of the primitive central idempotent in $H$ affording the irreducible representation $f_{\xi_{\lambda}}$ of $H$ of degree one, as in the statement of the theorem, by [Curtis and Reiner 1981, Corollary 11.26 and Theorem 11.25]. The last statement of the theorem also follows from [Curtis and Reiner 1981, Theorem 11.25].

Theorem 6.3. Let $\lambda$ be an irreducible character of $B$ with $U$ in its kernel. Let $\xi_{\lambda}$ be the irreducible character of $G$ which appears with multiplicity one in $\lambda^{G}$ and in the Gelfand-Graev character $\psi^{G}$, and let $f_{\xi_{\lambda}}: H \rightarrow \mathbb{C}$ be the irreducible representation of the Hecke algebra $H$ of the Gelfand-Graev representation $\psi^{G}$ defined in Theorem 6.2. There exists a unique homomorphism of algebras $f_{T}: H \rightarrow \mathbb{C} T$, independent of $\lambda$, such that, for each linear character $\lambda$ of $T$, one has

$$
f_{\xi_{\lambda}}(h)=\tilde{\lambda} \circ f_{T}(h), h \in H,
$$

where $\tilde{\lambda}$ is the extension of $\lambda: T \rightarrow \mathbb{C}$ to the group algebra $\mathbb{C} T$. The homomorphism $f_{T}$ is given by the formula $f_{T}\left(c_{n}\right)=\sum_{t \in T} f_{T}\left(c_{n}\right)(t) t$, where

$$
f_{T}\left(c_{n}\right)(t)=\operatorname{ind} n|B|^{-1}|U|^{-1} \sum_{g \in G, u \in U, g u n g^{-1}=t u^{\prime}} \psi\left(u^{-1}\right),
$$

for a standard basis element $c_{n}$ of $H$ and $g u n g^{-1}=t u^{\prime}, t \in T, u^{\prime} \in U$, is an element of $B$ which projects onto the element $t \in T$ by the homomorphism $B \rightarrow T$. If there are no solutions to the equation gung ${ }^{-1}=t u^{\prime}$, then $f_{T}\left(c_{n}\right)(t)=0$.

By the proof of Theorem 6.2, the representation $f_{\xi_{\lambda}}$ of the Hecke algebra $H$ is the restriction to $H$ of the unique irreducible character (see Lemma 6.1) $\xi_{\lambda}$ extended 
to the group algebra $\mathbb{C} G$. Moreover, the proof of Theorem 6.2 shows that

$$
f_{\xi_{\lambda}}(h)=\lambda^{G}(h), \quad h \in H,
$$

with $\lambda^{G}$ extended to the group algebra, because $e \boldsymbol{l}_{\lambda}=e \boldsymbol{x}_{\lambda}$ and $e \boldsymbol{x}_{\lambda}$ affords the representation $f_{\xi_{\lambda}}$ of $H=e \mathbb{C} G e \subseteq \mathbb{C} G$. In more detail, $h$ lies in $H$, and is viewed as an element of the group algebra $\mathbb{C} G$. Then $\lambda^{G}(h)$ is the trace of the action of $h$ on a module $M$ affording the induced character $\lambda^{G}$. As $h e=h$, the trace is computed on the module $e M$, which is one dimensional, and affords the representation $f_{\xi_{\lambda}}$ of $H$, by Theorem 6.2.

For a standard basis element $c_{n}$ of $H$, we have

$$
c_{n}=\text { ind } n \text { ene }=|U|^{-1} \sum_{u_{1} n u_{2} \in U n U} \psi\left(u_{1}^{-1} u_{2}^{-1}\right) u_{1} n u_{2},
$$

by [Curtis and Reiner 1981, Proposition 11.30(i)]. Then, with $\lambda^{G}$ extended to the group algebra, we obtain

$\lambda^{G}\left(c_{n}\right)=|U|^{-1} \sum_{u_{1} n u_{2} \in U n U} \psi\left(u_{1}^{-1} u_{2}^{-1}\right) \lambda^{G}\left(u_{1} n u_{2}\right)=$ ind $n|U|^{-1} \sum_{u \in U} \psi\left(u^{-1}\right) \lambda^{G}(u n)$.

We have used the fact that the double coset $U n U$ contains ind $n$ one sided cosets. For the induced character we have, by [Curtis and Reiner 1981, 10.3],

$$
\lambda^{G}(\text { un })=|B|^{-1} \sum_{g \in G} \dot{\lambda}\left(g^{-1} u n g\right),
$$

where $\dot{\lambda}(x)=0$ if $x \notin B$. Then $\dot{\lambda}\left(g^{-1} u n g\right) \neq 0$ only if $g^{-1} u n g=u^{\prime} t$ with $u^{\prime} \in U$ and $t \in T$, and in that case, $\dot{\lambda}\left(g^{-1} u n g\right)=\lambda(t)$. Therefore

$$
\lambda^{G}\left(c_{n}\right)=\operatorname{ind} n|B|^{-1}|U|^{-1} \sum_{t \in T} \sum_{g^{-1} \text { ung }=u^{\prime} t} \psi\left(u^{-1}\right) \lambda(t) .
$$

Then, for $t \in T$,

$$
f_{T}\left(c_{n}\right)(t)=\operatorname{ind} n|B|^{-1}|U|^{-1} \sum_{g^{-1} \text { ung }=u^{\prime} t} \psi\left(u^{-1}\right)
$$

is independent of $\lambda$, and we have

$$
f_{\xi_{\lambda}}(h)=\tilde{\lambda} \circ f_{T}(h), \quad h \in H .
$$

The facts that $f_{T}: H \rightarrow \mathbb{C} T$ is a homomorphism of algebras and is a uniquely determined linear map with the factorization property stated in the theorem both follow from the orthogonality relations for the linear characters $\lambda$ of $T$. This completes the proof of the theorem.

It is a nice exercise to derive the formula for the homomorphism $f_{T_{0}}: H \rightarrow \mathbb{C} T_{0}$ given in Theorem 5.2 from the statement of the preceding theorem. 
Theorem 6.3, for principal series representations of finite Chevalley groups, is a special case of Theorem 4.2 in [Curtis 1993] for representations $R_{T, \theta}$ of connected reductive algebraic groups defined over finite fields. The point of including it here is that in the special case of principal series representations, it is possible to give a combinatorial proof of the existence of the homomorphisms $f_{T}$.

\section{References}

[Bonnafé and Kessar 2008] C. Bonnafé and R. Kessar, "On the endomorphism algebras of modular Gelfand-Graev representations”, J. Algebra 320:7 (2008), 2847-2870. MR 2010f:20046 Zbl 1197.20037

[Borel and Tits 1972] A. Borel and J. Tits, "Compléments à l'article: "Groupes réductifs"”, Inst. Hautes Études Sci. Publ. Math. 41 (1972), 253-276. MR 47 \#3556 Zbl 0254.14018

[Chang 1976] B. Chang, "Decomposition of Gelfand-Graev characters of $\mathrm{LGL}_{3}(q)$ ", Comm. Algebra 4:4 (1976), 375-401. MR 53 \#5766 Zbl 0356.20051

[Chevalley 1955] C. Chevalley, "Sur certains groupes simples", Tôhoku Math. J. (2) 7 (1955), 14-66. MR 17,457c Zbl 0066.01503

[Curtis 1988] C. W. Curtis, "A further refinement of the Bruhat decomposition", Proc. Amer. Math. Soc. 102:1 (1988), 37-42. MR 89d:20038 Zbl 0656.20049

[Curtis 1993] C. W. Curtis, "On the Gelfand-Graev representations of a reductive group over a finite field”, J. Algebra 157:2 (1993), 517-533. MR 94i:20075 Zbl 0787.20026

[Curtis 2009] C. W. Curtis, "On problems concerning the Bruhat decomposition and structure constants of Hecke algebras of finite Chevalley groups", Michigan Math. J. 58:1 (2009), 213-230. MR 2011a:20007 Zbl 1241.20004

[Curtis and Reiner 1981] C. W. Curtis and I. Reiner, Methods of representation theory with applications to finite groups and orders, vol I., John Wiley \& Sons, New York, 1981. MR 82i:20001 Zbl 0469.20001

[Deligne and Lusztig 1976] P. Deligne and G. Lusztig, "Representations of reductive groups over finite fields", Ann. of Math. (2) 103:1 (1976), 103-161. MR 52 \#14076 Zbl 0336.20029

[Deodhar 1985] V. V. Deodhar, "On some geometric aspects of Bruhat orderings, I: a finer decomposition of Bruhat cells", Invent. Math. 79:3 (1985), 499-511. MR 86f:20045 Zbl 0563.14023

[Digne and Michel 1991] F. Digne and J. Michel, Representations of finite groups of Lie type, London Mathematical Society Student Texts 21, Cambridge University Press, Cambridge, 1991. MR 92g:20063 Zbl 0815.20014

[Gelfand and Graev 1962a] I. M. Gelfand and M. I. Graev, "Categories of group representations and the classification problem of irreducible representations", Dokl. Akad. Nauk SSSR 146 (1962), 757-760. In Russian; translated in Sov. Math. Dokl. 3 (1962), 1378-1381. MR 26 \#237

[Gelfand and Graev 1962b] I. M. Gelfand and M. I. Graev, "Construction of irreducible representations of simple algebraic groups over a finite field", Dokl. Akad. Nauk SSSR 147 (1962), 529-532. In Russian; translated in Sov. Math. Dokl. 3 (1962), 1646-1649. MR 26 \#6271 Zbl 0119.26902

[Iwahori and Matsumoto 1965] N. Iwahori and H. Matsumoto, "On some Bruhat decomposition and the structure of the Hecke rings of p-adic Chevalley groups", Inst. Hautes Études Sci. Publ. Math. 25 (1965), 5-48. MR 32 \#2486 Zbl 0228.20015

[Kawanaka 1975] N. Kawanaka, "Unipotent elements and characters of finite Chevalley groups", Osaka J. Math. 12:2 (1975), 523-554. MR 52 \#5784 Zbl 0314.20031 
[Kilmoyer 1978] R. W. Kilmoyer, "Principal series representations of finite Chevalley groups", $J$. Algebra 51:1 (1978), 300-319. MR 81e:20047 Zbl 0389.20008

[Simion 2015] I. I. Simion, "On refinements of the Bruhat decomposition", J. Algebraic Combin. (online publication August 2015).

[Steinberg 1968] R. Steinberg, "Lectures on Chevalley groups", mimeographed notes, Yale University, New Haven, 1968. MR 57 \#6215 Zbl 1196.22001

[Whittaker and Watson 1927] E. T. Whittaker and G. N. Watson, A course of modern analysis: an introduction to the general theory of infinite processes and of analytic functions; with an account of the principal transcendental functions, 4th ed., Cambridge University Press, Cambridge, 1927. MR 97k:01072 Zbl 53.0180.04

Received March 4, 2015. Revised July 24, 2015.

Charles W. CuRTis

Department of Mathematics And Institute of Theoretical SCIEnCE

UNIVERSITY OF OREGON

EUGENE, OR 97403

UNITED STATES

cwc@uoregon.edu 


\title{
PACIFIC JOURNAL OF MATHEMATICS
}

\author{
msp.org/pjm
}

Founded in 1951 by E. F. Beckenbach (1906-1982) and F. Wolf (1904-1989)

\section{EDITORS}

Don Blasius (Managing Editor)

Department of Mathematics

University of California

Los Angeles, CA 90095-1555

blasius@math.ucla.edu

\author{
Paul Balmer \\ Department of Mathematics \\ University of California \\ Los Angeles, CA 90095-1555 \\ balmer@math.ucla.edu \\ Robert Finn \\ Department of Mathematics \\ Stanford University \\ Stanford, CA 94305-2125 \\ finn@math.stanford.edu \\ Sorin Popa \\ Department of Mathematics \\ University of California \\ Los Angeles, CA 90095-1555 \\ popa@math.ucla.edu
}

\author{
Vyjayanthi Chari \\ Department of Mathematics \\ University of California \\ Riverside, CA 92521-0135 \\ chari@math.ucr.edu \\ Kefeng Liu \\ Department of Mathematics \\ University of California \\ Los Angeles, CA 90095-1555 \\ liu@math.ucla.edu \\ Jie Qing \\ Department of Mathematics \\ University of California \\ Santa Cruz, CA 95064 \\ qing@ cats.ucsc.edu
}

\section{PRODUCTION}

Silvio Levy, Scientific Editor, production@msp.org

\section{SUPPORTING INSTITUTIONS}

ACADEMIA SINICA, TAIPEI

CALIFORNIA INST. OF TECHNOLOGY

INST. DE MATEMÁTICA PURA E APLICADA

KEIO UNIVERSITY

MATH. SCIENCES RESEARCH INSTITUTE

NEW MEXICO STATE UNIV.

OREGON STATE UNIV.

\author{
STANFORD UNIVERSITY \\ UNIV. OF BRITISH COLUMBIA \\ UNIV. OF CALIFORNIA, BERKELEY \\ UNIV. OF CALIFORNIA, DAVIS \\ UNIV. OF CALIFORNIA, LOS ANGELES \\ UNIV. OF CALIFORNIA, RIVERSIDE \\ UNIV. OF CALIFORNIA, SAN DIEGO \\ UNIV. OF CALIF., SANTA BARBARA
}

\author{
Daryl Cooper \\ Department of Mathematics \\ University of California \\ Santa Barbara, CA 93106-3080 \\ cooper@math.ucsb.edu \\ Jiang-Hua Lu \\ Department of Mathematics \\ The University of Hong Kong \\ Pokfulam Rd., Hong Kong \\ jhlu@maths.hku.hk \\ Paul Yang \\ Department of Mathematics \\ Princeton University \\ Princeton NJ 08544-1000 \\ yang@math.princeton.edu
}

These supporting institutions contribute to the cost of publication of this Journal, but they are not owners or publishers and have no responsibility for its contents or policies.

See inside back cover or msp.org/pjm for submission instructions.

The subscription price for 2015 is US \$420/year for the electronic version, and \$570/year for print and electronic.

Subscriptions, requests for back issues and changes of subscribers address should be sent to Pacific Journal of Mathematics, P.O. Box 4163, Berkeley, CA 94704-0163, U.S.A. The Pacific Journal of Mathematics is indexed by Mathematical Reviews, Zentralblatt MATH, PASCAL CNRS Index, Referativnyi Zhurnal, Current Mathematical Publications and Web of Knowledge (Science Citation Index).

The Pacific Journal of Mathematics (ISSN 0030-8730) at the University of California, c/o Department of Mathematics, 798 Evans Hall \#3840, Berkeley, CA 94720-3840, is published twelve times a year. Periodical rate postage paid at Berkeley, CA 94704, and additional mailing offices. POSTMASTER: send address changes to Pacific Journal of Mathematics, P.O. Box 4163, Berkeley, CA 94704-0163.

PJM peer review and production are managed by EditFLOW ${ }^{\circledR}$ from Mathematical Sciences Publishers.

\section{PUBLISHED BY}

\section{mathematical sciences publishers \\ nonprofit scientific publishing}

http://msp.org/

(C) 2015 Mathematical Sciences Publishers 


\title{
PACIFIC JOURNAL OF MATHEMATICS
}

\author{
Volume 279 No. 1-2 December 2015
}

In memoriam: Robert Steinberg

Robert Steinberg (1922-2014): In memoriam V. S. VARADARAJAN

Cellularity of certain quantum endomorphism algebras

HENNING H. ANDERSEN, GUSTAV I. LEHRER and RUIBIN ZHANG

Lower bounds for essential dimensions in characteristic 2 via orthogonal representations ANTONIO BABIC and VLADIMIR CHERNOUSOV

Cocharacter-closure and spherical buildings

Michael Bate, Sebastian Herpel, Benjamin Martin and Gerhard RöHrLe

Embedding functor for classical groups and Brauer-Manin obstruction

Eva Bayer-Fluckiger, Ting-Yu LeE and Raman Parimala

On maximal tori of algebraic groups of type $G_{2}$

Constantin Beli, PhilipPe Gille and Ting-Yu LeE

On extensions of algebraic groups with finite quotient

MICHEL BRION

Essential dimension and error-correcting codes

SHANE CERNELE and ZiNOVy REICHSTEIN

Notes on the structure constants of Hecke algebras of induced representations of finite Chevalley groups

Charles W. CuRTis

Complements on disconnected reductive groups

FRANÇOIS DIGNE and JEAN MICHEL

Extending Hecke endomorphism algebras

Jie Du, Brian J. Parshall and LeOnard L. SCOTT

Products of partial normal subgroups

ELLEN HENKE

Lusztig induction and $\ell$-blocks of finite reductive groups

RADHA KESSAR and GUNTER MALLE

Free resolutions of some Schubert singularities

Manoj Kummini, Venkatramani Lakshmibai, Pramathanath Sastry and C. S. Seshadri

Free resolutions of some Schubert singularities in the Lagrangian Grassmannian

VenKatramani LAKSHMibai and ReUVEN HODGES

Distinguished unipotent elements and multiplicity-free subgroups of simple algebraic groups

Martin W. Liebeck, Gary M. Seitz and Donna M. Testerman

Action of longest element on a Hecke algebra cell module

GEORGE LUSZTIG

Generic stabilisers for actions of reductive groups

BENJAMIN MARTIN

On the equations defining affine algebraic groups

VLADIMIR L. POPOV

Smooth representations and Hecke modules in characteristic $p$

PETER SCHNEIDER

On CRDAHA and finite general linear and unitary groups

BHAMA SRINIVASAN

Weil representations of finite general linear groups and finite special linear groups PHAM HUU TIEP

The pro- $p$ Iwahori Hecke algebra of a reductive $p$-adic group, $\mathrm{V}$ (parabolic induction) MARIE-FRANCE VIGNÉRAS

Acknowledgement 